auf Kathodenpotential läge. In diesem Fall lassen sich die Sondenvorstellungen ganz analog anwenden. Man muß hier aber bedenken, daß auch die Austrittsblende (A) negative Sonde im Plasma ist und einen positiven Ionenstrom aufnimmt. Dann tritt eine Stromverteilung zwischen A und dem herausgezogenen Bündelstrom $\left(J_{\mathrm{F}}\right)$ auf, welche ebenfalls ein "Raumladungsgesetz“ ( $\left.J_{\mathrm{F}} \sim U^{3 / 2}\right)$ ergeben kann.

$15 \mathrm{~J}$. Kistemaker u. H. L. Douwes Dek ker, Physica 16, 198 u. 209 [1950].

\title{
Massenspektrometrische Untersuchungen der Feldemission positiver lonen
}

\author{
Von Mark G. Inghram und Robert Gomer \\ Aus dem Department of Physics, University of Chicago, Chicago, Illinois, \\ und Argonne National Laboratory, Lemont, Illinois, USA \\ und dem Department of Chemistry, und Institute for the Study of Metals, \\ University of Chicago, Chicago, Illinois, USA \\ (Z. Naturforschg. 10 a, 863-872 [1955]; eingegangen am 2. August 1955) \\ Herrn Professor Dr. J. Mattauch zum 60. Geburtstag gewidmet
}

\begin{abstract}
Massenspektrometrische Untersuchungen der in der Nähe und an der Oberfläche einer Spitze eines Feld-Emissions-Mikroskops (FEM) erzeugten positiven Ionen erläutern den Mechanismus von Ionisierungsvorgängen und weisen auf verschiedene Anwendungen hin. Letztere bestehen in einer Bestimmung der Dissoziationsprodukte bei Chemisorption, Analyse von Gasmischungen, Nachweis und Messung kurzlebiger Zwischenprodukte, freier Radikale und so weiter.
\end{abstract}

$\mathrm{D}$ e Desorption positiver Ionen von Oberflächen unter Einwirkung starker elektrischer Felder wurde zuerst von $\mathrm{M} \ddot{\mathrm{u} l l} \mathrm{er}$ beobachtet ${ }^{1}$. Er fand, daß Schichten von Barium, Strontium, Natrium und anderen elektropositiven Metallen bei Feldstärken von 0,5 bis $2,0 \mathrm{Volt} / \AA$ von positiv geladenen FEMSpitzen abgerissen wurden. In späteren Untersuchungen benutzte Müller eine mit Wasserstoff von einem Druck von $10^{-3}$ Torr gefüllte Feldemissionsröhre und zeigte, daß bei positiver Ladung der Spitze $(F=2$ Volt $/ \AA)$ ein schwaches, aber äußerst scharfes Bild entstand, welches er der Desorption von Protonen zuschrieb ${ }^{1}$.

Der ursprüngliche Zweck der vorliegenden Arbeit war die Bestimmung der Ionen, die von starken Feldern erzeugt werden können. Zu diesem Zweck ließen wir einen kleinen Teil des Ionenstrahls von einer FEM-Spitze durch ein feines Loch (ca. 0,75 $\mathrm{mm}$ ) im Schirm einer Feldemissionsröhre in den Analysator eines Massenspektrometers eindringen. Die Feldemissionsanordnung diente also als Ionenquelle in einem sonst herkömmlichen Massenspektrometer.

Es erwies sich, daß Ionen von allen gasförmigen Substanzen erzeugt werden konnten, unabhängig davon, ob diese elektropositiv oder elektronegativ, fest

1 E. W. M üller, Erg. exakt. Naturwiss. 27, 290 [1953].

2 M. G. I n h r a m u. R. G o m er, J. Chem. Phys. 22, 1279 [1954]. oder gar nicht adsorbiert waren. Es zeigte sich auch bald, daß die Verbindung von Massenspektrometrie mit Feldionisierung interessante Möglichkeiten darbietet für die Untersuchung von Adsorptionsvorgängen, für Gasanalysen und für den Nachweis sowie die Messung von kurzlebigen oder schwachen Reaktionsprodukten. Die Untersuchungen, über die hier berichtet werden soll ${ }^{2,3}$, stellen zwar nur einen Anfang dar, scheinen aber doch einer genaueren Beschreibung wert zu sein.

\section{Versuchsanordnung}

Die Ionenquelle und der erste Teil des Massenspektrometers sind in Abb. 1 gezeigt. Das Emissionsmikroskop wurde mittels einer „Kovar“-Glas-Metall-Verbindung von $2,5 \mathrm{~cm} \phi$ an einem zylindrischen Stück von „Inconel"-Metall angebracht. Dieses Stück wurde mit zwölf Bolzen von rund $0,8 \mathrm{~cm}$ Durchmesser am Massenspektrometer befestigt. Gasdichte wurde durch eine toroidale Gold-Dichtung von $0,064 \mathrm{~cm}$ Durchmesser erreicht. Ein Inconel-Einsatz mit einem Loch von $0,102 \mathrm{~cm} \varnothing$ ragte vom Massenspektrometer bis fast zur Rückseite des Leuchtschirms vor. Die FEM-Spitze und das Austrittsloch im Schirm wurden hinsichtlich des Massenspektrometers sorgfältig justiert.

Gasproben konnten durch das Pumprohr des Emissionsmikroskops mittels ausheizbarer Metallventile und veränderlicher Spalte aus Vorratsgefäßen eingeführt werden. Alle

3 R. Gom e r u. M. G. In g h ram, J. Amer. Chem. Soc. 77, 500 [1955]. 
Bestandteile des Apparats, wie Vorratssystem, Emissionsröhre, Analysator und Detektor, waren mit eigenen Diffusionspumpen versehen.

Im Gebrauch wurde der Apparat zuerst auf einen Anfangsdruck von rund $10^{-8}$ Torr evakuiert, sodann die Spitze entgast und ein Elektronenbild aufgenommen, so daß die Orientierung der Spitze bestimmt werden konnte. Aus FowlerNordheim-Kennlinien für reine Spitzen oder solche von bekannter Bedeckung konnte das Oberflächenfeld und der Spitzendurchmesser ungefähr ermittelt werden. Das zu untersuchende Gas wurde dann bis zum gewünschten Druck in die Emissionsröhre eingelassen und die Spitze auf Ionenemission umgeschaltet.

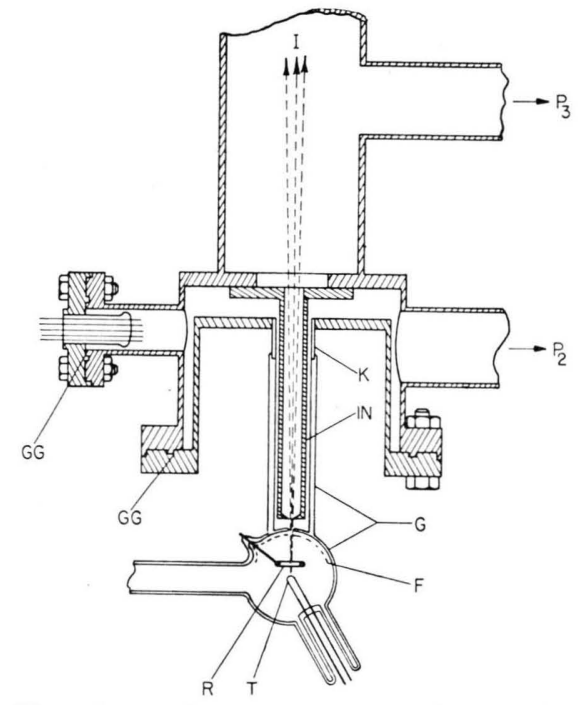

Abb. 1. Versuchsanordnung zur massenspektrometrischen Untersuchung der Feldemission positiver Ionen: Ionenquelle und erste Stufe des Spektrometers. Die FEM-Anlage kann abgenommen und durch Elektronenstoß- oder andere Ionenquellen ersetzt werden. Die FEM-Röhre ist nur schematisch gezeichnet. (Beobachtungsfenster und Bestrahlungsfenster aus Quarz nicht gezeigt.)

\section{Emissionsröhre}

Die Emissionsröhre ist schematisch in Abb. 1 gezeigt und unterscheidet sich von üblichen Röhren nur dadurch, daß das Bild von der rückwärtigen Seite durch ein flaches Glasfenster beobachtet werden muß. Die Zuleitungsdrähte und der Anodenring stören dabei nur wenig. Der Leuchtschirın bestand aus Zinksilikat und wurde auf elektrisch leitendem Glas $^{4}$ niedergeschlagen. Einige Sorgfalt wurde angewandt, um die Wände des Austrittsloches ebenfalls leitfähig zu machen. Der Anodenring bestand aus $0,50 \mathrm{~mm}$ dickem Wolframdraht und war fast bis zum ringförmigen Teil mit NonexGlas überzogen. Elektrische Verbindung mit dem Schirm wurde durch einen Federkontakt aus dünnem Wolframdraht hergestellt. Die Emissionspitze befand sich fast in der Ebene des Anodenringes. Wie schon von $\mathrm{M}$ ülle $\mathrm{r}^{1}$ angedeutet wurde, ermöglicht die Verkleinerung des Beschleunigungs-

${ }^{4}$ R. G o m e r, Rev. Sci. Instrum. 24, 993 [1953]. raumes die Verwendung von höheren Gasdrucken (das heißt kürzeren freien Weglängen), ohne eine Gasentladung hervorzurufen. Der Anodenring verhindert auch die Feldemission von Elektronen vom Leuchtschirm, wenn die Spitze positiv geladen ist.

Die Spitzenanordnung besteht aus einer Wolframdrahtschleife von $0,25 \mathrm{~mm}$ Dicke, an die der Spitzendraht geschweißt ist. Zwei Spannungsmeßdrähte von $0,075 \mathrm{~mm}$ Dicke, ebenfalls aus Wolfram, sind $1 \mathrm{~cm}$ vom Scheitel der Schleife angeschweißt, so daß die Spitzentemperatur aus dem elektrischen Widerstand der Schleife bestimmt werden kann. Die Justierung der Spitze wurde folgendermaßen vorgenommen: Eine frisch geätzte Spitze wurde in die abmontierte FEMAnlage eingeschmolzen und so gut wie möglich auf den Mittelpunkt des Anodenringes gerichtet. Eine flache Aluminiumscheibe mit zylindrischem Aufsatz wurde sodann an den Inconel-Zylinder angelegt, so daß der Aufsatz in das KovarGlasrohr eindrang und eine ähnliche Lage wie der InconelEinsatz in Betrieb einnahm. Ein Loch in der Drehachse des Aluminiumstückes erlaubte die Einführung eines Stahlstabes, an dessen Ende ein feiner, gespitzter Stab von $0,51 \mathrm{~mm} \phi$ koaxial angebracht war. Dieser Stab konnte durch das Austrittsloch bis knapp zur FEM-Spitze in die Emissionsröhre eingeführt werden. Es war dann relativ einfach, die Spitze zu justieren, indem die gläserne Einschmelzröhre erhitzt und so lange manipuliert wurde, bis die Spitze und der Stab aus zwei gegeneinander senkrechten Richtungen koplanar erschienen.

Die in Abb. 1 gezeichnete Versuchsanordnung erlaubt die Untersuchung der Ionenemission von kleinen Teilen einzelner Kristallflächen der Spitze. Wenn kristallographische Anisotropie keine Rolle spielt, kann die Ionenintensität durch einen fokussierenden Aufbau stark gesteigert werden. Eine Vorrichtung dieser Art (elektrostatisches Linsensystem) läßt $20 \%$ der emittierenden Fläche als scheinbare Quelle erscheinen. Die Intensitätssteigerung beruht nicht nur auf geometrischen Gründen, sondern kommt auch von der großen Emissionsanisotropie verschiedener Kristallflächen her, deren Lage bezüglich des Analysators mehr oder weniger vom Zufall bedingt ist. Es ist möglich, eine bewegliche Spitze zu bauen, doch haben wir das noch nicht durchgeführt. Weiter ist es möglich, die Intensität dadurch zu steigern, daß eine Spitze aus Pintschdraht [in Richtung der (111)-Achse orientiert] benutzt wird, da die (111)-Richtung eine hohe Intensität ergibt.

\section{Massenspektrometer}

Das Massenspektrometer war ein richtungsfokussierendes $60^{\circ}$-Sektorfeld-Instrument von $30,5 \mathrm{~cm}$ Radius mit einem Elektronenvervielfacher als Detektor. Das Grundmaterial der Konstruktion war Inconel-Metall. Das Pumpensystem bestand aus vier Quecksilber-Diffusionspumpen, drei für das Massenspektrometer und eine für das Gasreservoir. Mechanische Ölpumpen (Welch Duo Seal) sorgten für das Vorvakuum. Das Vorratssystem und die Emissionsröhre waren mit BayardAlpert-Dünndrahtanoden-Ionisierungsmanometern ${ }^{5}$ (Typ Westinghouse 1949) und Alpert-Kupferfallen ${ }^{5}$ versehen, die auf $400^{\circ} \mathrm{C}$ ausgeheizt werden konnten.

5 D. A l pe rt, J. Appl. Phys. 24, 860 [1953]; Rev. Sci. Instrum. 24, 1004 [1953]. 
Alle ständigen Metallverbindungen waren in einer HeliumAtmosphäre geschweißt worden. Als elektrische Zuführungsdrähte dienten in Glas eingeschmolzene Wolframstäbe. Alle abnehmbaren Verbindungen wurden mit $0,64 \mathrm{~mm}$ dicken ringförmigen Golddrähten $(24 \mathrm{Kt}$.) gedichtet. Da solche Dichtungen ungefähr 20-mal an- und abmontiert werden können und die Kosten der Wiederherstellung nur rund $15 \%$ des Originalpreises betragen, macht sich die Benutzung von Gold bezahlt. Die ganze Apparatur ist daher frei von Gummi, Fetten und Metallen hohen Dampfdrucks, die in Silberlot vorkommen. Die Spektrometerröhre kann bis zu $425^{\circ} \mathrm{C}$ ausgeheizt werden, so daß Hochvakuum erreichbar ist. In den Vorversuchen, die hier beschrieben werden, wurden diese Möglichkeiten der Maschine noch nicht voll ausgenutzt.

Während der Feldemission positiver Ionen hat man den hohen Druck (oft $5 \cdot 10^{-3}$ Torr) in der Emissionsröhre zu bekämpfen. Drucke dieser Größenordnung würden wesentliche Streufehler im Analysator und Entladungen im Elektronenvervielfacher hervorrufen. Um diese Störungen zu verhindern, wurden alle Stufen der Maschine einzeln ausgepumpt. Die Drucke beiderseits des Austrittsloches in der Emissionsröhre unterscheiden sich ca. um den Faktor 100, beiderseits des Inconel-Einsatzes ebenfalls um den Faktor 100 , so daß Drucke in der Röhre von $5 \cdot 10^{-3}$ Torr nur $5 \cdot 10^{-7}$ Torr im Analysator entsprachen; das genügte, um gutes Auflösungsvermögen und Vervielfacherstabilität zu erhalten.

Abb. 2 zeigt das Schaltbild für Ionenerzeugung. $E_{1}$ ist dabei die Beschleunigungsspannung, welche die Ionenenergie im magnetischen Analysatorfeld bestimmt. $E_{2}$ kann null sein oder einer konstanten oder pulsierenden Spannung entsprechen. Der Kondensator $C$ hält die Spannung zwischen Spitze und Analysator bei Pulsbetrieb konstant. $E_{2}$ kann unabhängig von $E_{1}$ verändert werden, so daß Verschiebungen im magnetischen Analysatorfeld bei Veränderungen der Entstehungsspannung nicht nötig sind. Verlangsamung der Ionen durch entsprechende Kombination von $E_{1}$ und $E_{2}$ ergibt bessere Energieauflösung, als mit $E_{2}=0$ erhältlich ist. Der Spannungspulsator, der in einigen Versuchen verwendet

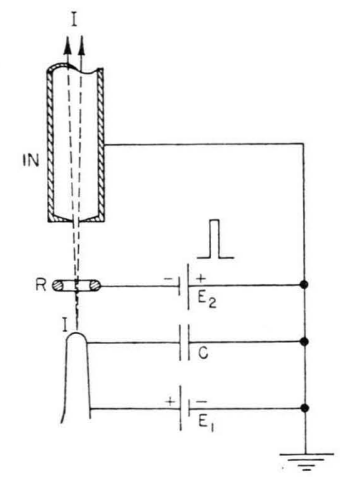

Abb. 2. Schaltungsschema für Ionenemission. $E_{1}$ Beschleunigungsspannung für das Massenspektrometer. $E_{1}+E_{2}$ Entstehungsspannung. $E_{2}$ kann eine Gleich- oder pulsierende Spannung sein.

${ }^{6}$ R. G o mer u. R. W ortman, J. Chem. Phys. 23, 1741 [1955]. wurde, gab 10000 -Volt-Pulse von $2 \mu$ sec Dauer und einer „rise time“" von 0,1 usec.

\section{Ergebnisse und Mechanismus}

Alle hier beschriebenen Experimente wurden mit einer Wolframspitze ausgeführt. Das Massenspektrometer sah gewöhnlich einen kleinen Teil der (110)-Fläche von ca. $400 \AA^{2}$. Die genaue Größe des Flächeninhalts hängt vom Radius der Spitze ab. In fast allen Versuchen war der Anfangsdruck $10^{-8}$ Torr, so daß eine Sauerstoffvergiftung der Spitze nicht vermieden werden konnte. Diese Tatsache spielt eine gewisse Rolle, wie sich herausstellen wird. Die mit den untersuchten Gasen erhaltenen Resultate sind in Tab. $2 *$ kurz zusammengefaßt. Die relativen Ionenausbeuten sind nicht konstant, sondern hängen vom Druck, der Temperatur, der Feldstärke und dem Zustand der Spitze ab. Die Tabelle gibt daher nur typische Werte an. Die Feldstärken, bei denen Ionisierung eintritt, sind früher ${ }^{2}$ höher angegeben als hier. Die Werte wurden damals nach der Fowler-Nordheim-Gleichung mit einer Austrittsarbeit von 6,2 Volt berechnet. Diese entspricht einer mit Sauerstoff bedeckten Spitze und wurde wegen des relativ schlechten Vakuums als die richtige angesehen, obwohl sich Wasserstoff im Massenspektrometer befand. Im Verlauf einer anderen Arbeit ${ }^{6}$ wurde vor kurzem festgestellt, daß die gemischte Adsorption von Sauerstoff und Wasserstoff zu Austrittsarbeiten von ungefähr 4,5 Volt führt, wenn der Wasserstoff zuerst adsorbiert wird. Die früher ${ }^{2}$ gefundenen Feldwerte sind daher um rund 1,7 zu hoch.

\section{Schwache Adsorption}

Bei den in Tab. $2^{*}$ angegebenen Resultaten ist am auffälligsten, daß Masseneffekte bei der Ionisierung nur eine geringe Rolle spielen. Auch ist es unwahrscheinlich, daß Moleküle wie $\mathrm{H}_{2}, \mathrm{O}_{2}, \mathrm{~N}_{2}, \mathrm{CH}_{4}$ usw. ohne Zersetzung stark adsorbiert werden. Diese Überlegungen machen einen Tunneleffekt für positive Ionen unwahrscheinlich. Hingegen ist anzunehmen, daß Ionen durch Feldemission von Elektronen aus nahe der Spitze befindlichen Molekülen entstehen ${ }^{2,7}$. Bei relativ geringen Feldstärken sollte dies häufiger in der unmittelbaren Nähe der Spitze als im freien Raum stattfinden, weil Bild- und Austausch-Kräfte den von den Elektronen zu durchdringenden Potentialberg herabsetzen. Abb. 3 zeigt den Potentialverlauf für die angenommenen Fälle von Wasserstoff-Atomen im 1s-Zustand nahe einer Wolframoberfläche und im freien Raum unter der Einwirkung eines elektrischen Feldes von 2 Volt/A. Bei genügender Feldstärke sollten die Bild- und Austausch-Effekte wegfallen und reine Feldionisation

* Tab. 2. auf S. 870 .

7 F. Kirchner, Naturwiss. 6, 136 [1954]. 

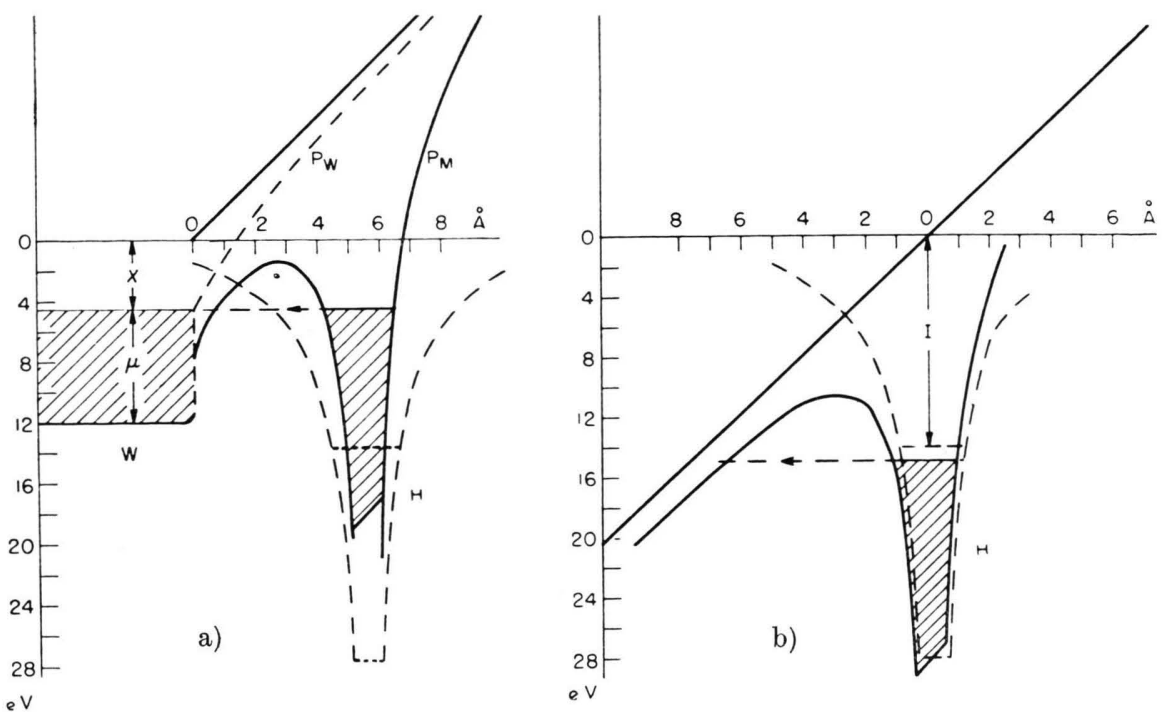

Abb.3.Mechanismus der Feldionisierung. a) Ionisierung eines Wasserstoffatoms in der Nähe einer Wolfram-Oberfläche. b) Derselbe Vorgang im freien Raum. $F$ Feldstärke $(F=2 \mathrm{~V} / \AA)$, W Wolfram, H Wasserstoffatom, $P_{\mathrm{W}}$ Bildkraftpotential, vom Feld verzerrt, $P_{\mathrm{M}}$ Potentialkurve des Wasserstoffatoms, vom Feld verzerrt, $I$ gleich IP von Wasserstoffatomen, $X$ Austrittsarbeit, $\mu$ Fermi-Niveau. Gestrichelte Kurven zeigen den Potentialverlauf in Abwesenheit des Feldes. stattfinden. Diese qualitativen Überlegungen werden vom Versuch gestützt. Abb. 4 zeigt das charakteristische Verhalten der Energieverteilung der meisten Ionen mit zunehmendem Felde. Man sieht, daß bei geringen Feldstärken die Schärfe der Ionenprofile nur durch das Auflösungsvermögen des Massenspektrometers beschränkt ist. Bei höheren Feldern entwickelt sich ein Schweif auf der Seite kleiner Energien, während bei den höchsten Feldstärken das
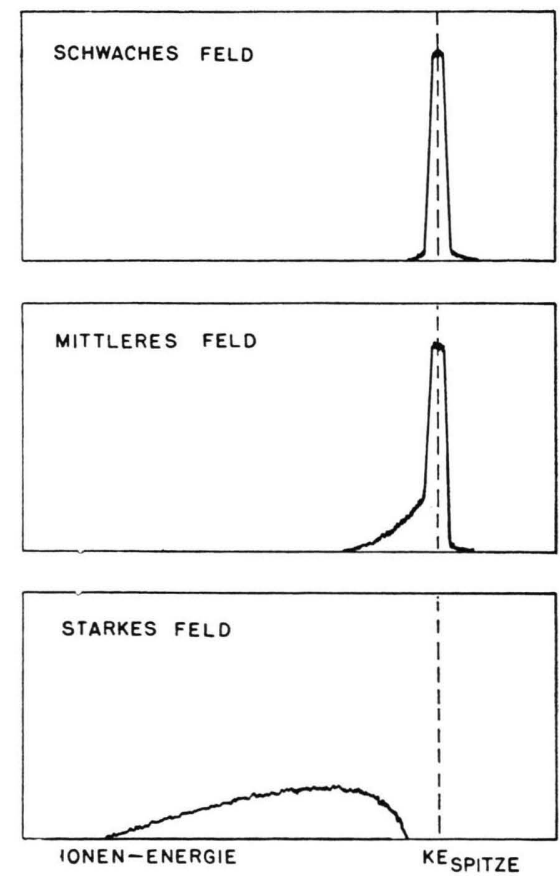

Abb. 4. Ionenprofile, die man bei schwach oder gar nicht adsorbierten Teilchen erhält, als Funktion der Feldstärke.
Profil sehr ausgebreitet und schon vor dem Einsetzen des ursprünglichen Profils auf Null abgefallen zu sein scheint. Da die größte Energie eines Ions der Spannung $E_{1}$ entspricht, muß eine Energieabfall einer Ionenerzeugung vor der Spitze entsprechen. So läßt es sich zeigen, daß die Ionisation bei hohen Feldstärken $100 \AA$ vor der Spitze stattfindet. In diesem Fall dient die Spitze nur dazu, das Feld hervorzurufen.

Während man Ionisierungswahrscheinlichkeiten für komplizierte Moleküle oder Atome nicht gut berechnen kann, lassen sich doch einige allgemeine Betrachtungen anstellen.

Der Ionenstrom, den man aus einem Raumelement zu erwarten hat, ist durch

$$
i=q n^{\prime}\left(1-e^{-t / \tau}\right)
$$

gegeben, wo $\tau$ die Halbwertszeit, $n^{\prime}$ die Zahl der im Felde eintreffenden Molekeln, $q$ die Ionenladung und $t$ die Zeit bedeutet, die ein Teilchen im Feld verbringen würde, wenn keine Ionisierung stattfände. $\mathrm{M} \ddot{\mathrm{u}} \mathrm{ll}$ e $\mathrm{r}$ hat darauf hingewiesen, daß $n^{\prime}$ 10- bis 50-mal größer als die gaskinetische Stoßzahl sein kann, da die Spitze polarisierte Atome und Molekeln anzieht. Bei niedrigem Feld geht Gl. (1) in

$$
i=q n^{\prime} t / \tau=q n_{0}{ }^{\prime} v / \tau
$$

über, wo $n_{0}{ }^{\prime}$ die Gasdichte in $\mathrm{Mol} / \mathrm{cm}^{3}$ und $v$ der Rauminhalt der Feldregion ist. Man sieht, daß Gl. (2) eine Reaktion mit der Geschwindigkeitskonstanten $1 / \tau$ darstellt. Die Dichte $n_{0}{ }^{\prime}$ ist aus derjenigen beim Feld null mittels folgender Gleichung:

$$
n_{0}{ }^{\prime}=n_{0} \exp \left(E_{\mathrm{f}} / k T\right)
$$


zu berechnen, wo $E_{\mathrm{f}}$ die Einwirkung vom Feld auf permanente oder induzierte Dipole darstellt und durch

$$
E_{\mathrm{f}}=P F+\frac{1}{2} \alpha F^{2}
$$

gegeben ist. $P$ ist dabei das Dipolmoment und $\alpha$ die Polarisierbarkeit. Bei hohem Felde geht Gl. (1) in

$$
i=q n^{\prime}
$$

über. $n^{\prime}$ kann man nun auf folgende Weise abschätzen: Die Feldstärke nahe der Spitze ist annähernd durch

$$
F=F_{0}\left(r_{\mathrm{t}} / r\right)^{2}
$$

gegeben. $F$ bedeutet die Feldstärke in einer Entfernung $r$ vom Zentrum der Spitze und $F_{0}$ die Feldstärke an der Spitzenoberfläche, wo $r=r_{\mathrm{t}}$. Man sieht aus den Gln. (4) und (6), daß die Einwirkung der Spitze auf Moleküle einer Zentralkraft entspricht. Daher ergibt sich

$$
\mathrm{d} r / \mathrm{d} t=(2 / m)^{1 / 2}\left(E-V^{\prime}\right)^{1 / 2},
$$

wo $m$ die Masse, $E$ die Gesamt- und $V^{\prime}$ die virtuelle potentielle Energie bedeutet. $E$ ist gleich $k T$. $V^{\prime}$ ist durch

$$
V^{\prime}=-E_{\mathrm{f}}+E(\varrho / r)^{2}
$$

gegeben, wo $\varrho$ die kleinste Entfernung des Teilchens von der Spitze ohne Feld bedeutet. Setzt man Gl. (8) in Gl. (7) ein und verlangt, $\mathrm{da} \beta \mathrm{d} r / \mathrm{d} t$ reell bleibe, so erhält man den Maximalwert von $\varrho$, der mit streifenden Teilchenbahnen zu vereinbaren ist:

$$
\varrho^{2}=r_{\mathrm{t}}^{2}\left(1+E_{\mathrm{f}} / k T\right) .
$$

Der Ausdruck $\left(1+E_{\mathrm{f}} / k T\right)$ stellt daher den zur Berechnung des Stromes notwendigen Vergrößerungsfaktor dar. Es ist zu Gl. (3) zu bemerken, daß $n_{0}{ }^{\prime} / n_{0}$ bei geringen Feldstärken in $n^{\prime} / n$ übergeht. Gl. (9) ist natürlich nur annähernd richtig, da die Spitze nicht als freie Kugel im Raum schwebt. Auch sind im Falle von permanenten Dipolmomenten nur ca. die Hälfte der Molekeln in Feldrichtung orientiert. Die andere Hälfte präzessiert um die Feldrichtung und wird von der Spitze abgestoßen.

Der Ionenstrom läßt sich aus den Gln. (1) bis (9) errechnen, wenn man $t$ und $\tau$ kennt. $t$ kann aus gaskinetischen Gesetzen und aus Gl. (7) abgeschätzt werden und beträgt ungefähr $10^{-10} \mathrm{bis} 10^{-11} \mathrm{sec}$ für die ganze Spitze. $\tau$ kann man nur in den einfachsten Fällen auch nur annähernd genau berechnen. Die

${ }^{8}$ R. H. Fow le r u. L. W. Nordheim, Proc. Roy. Soc., Lond. A 119, 173 [1928].
Durchlässigkeit $D$ einer Potentialschwelle ist ungefähr durch

$$
D=\exp \left(-6,84 \cdot 10^{7} \int \sqrt{V-E} \mathrm{~d} x\right)
$$

gegeben ${ }^{8}$, wo $V$ und $E$ die potentielle und kinetische Energie in $\mathrm{eV}$ bedeuten, und das Integral über den Verlauf der Schwelle in $\mathrm{cm}$ genommen wird. Die Frequenz $v$, mit der Elektronen aus dem Innern eines Atoms oder Moleküls an der Schwelle eintreffen, ist durch

$$
v=p / m_{\mathrm{e}}
$$

gegeben, wo $p$ der Impuls und $m_{\mathrm{e}}$ die Masse eines Elektrons im Inneren des Teilchens sind. $\lambda$ ist der Erwartungswert des Durchmessers der Elektronenbahn. Die Halbwertszeit $\tau$ ist dann

$$
\tau=1 / D v .
$$

Im allgemeinen kann man $v$ nur auf $10^{15}$ bis $10^{16} \mathrm{sec}^{-1}$ abschätzen und die zur Auswertung von Gl. (10) notwendigen Potentialkurven nicht zeichnen, vor allem, weil durch Polarisierung das effektive Feld geschwächt wird. Gl. (10) kann zur Abschätzung von $D$ durch

$$
\tau=10^{-15} \exp \left(+0,684 I^{3 / 2} / F\right)
$$

ersetzt werden ${ }^{9}$, wo $I$ das Ionenpotential in $\mathrm{eV}$ und $F$ die Feldstärke in $\mathrm{V} / \AA$ bedeutet.

\begin{tabular}{|c|c|c|c|}
\hline$F$ & $\tau_{1}$ & $\tau_{2}$ & $\tau_{1} / \tau_{2}$ \\
Volt $/ \AA$ & $\sec$ & sec & \\
\hline 0,5 & $1,3 \cdot 10^{-1}$ & $4 \cdot 10^{-4}$ & 300 \\
1,0 & $1,6 \cdot 10^{-10}$ & $1,2 \cdot 10^{-12}$ & 130 \\
1.5 & $1,6 \cdot 10^{-13}$ & $8 \cdot 10^{-15}$ & 20 \\
2,0 & $1,7 \cdot 10^{-14}$ & $2 \cdot 10^{-15}$ & 8 \\
& $2 \cdot 10^{-16}$ & $2 \cdot 10^{-16}$ & 1 \\
\hline
\end{tabular}

Tab. 1. Halbwertszeiten von Wasserstoffatomen bei hohen Feldstärken. $\tau_{1}$ bedeutet die Halbwertszeit freier Wasserstoffatome, $\tau_{2}$ die Halbwertszeit solcher Atome, die sich $9,5 / F \AA$ vor einer Wolframoberfläche befinden.

Im Falle von Wasserstoffatomen gelten Gl. (10) und (11) ziemlich genau. Tab. 1 gibt die Halbwertszeiten für freie Atome und solche, die sich

$$
d=(I-X) / F=9,5 / F \mathrm{~cm}
$$

von einer Wolframoberfläche befinden (angenommene Austrittsarbeit $X=4,5 \mathrm{eV}$ ). Diese Distanz entspricht bei gegebener Feldstärke der kleinsten Entfernung, bei der das durchdringende Elektron in das Metall eintreten darf.

9 Eine einfache Ableitung dieser Gleichung aus Gl. (10) ist zu finden bei R. Go m e r, J. Chem. Phys. 21, 1869 [1953]. 


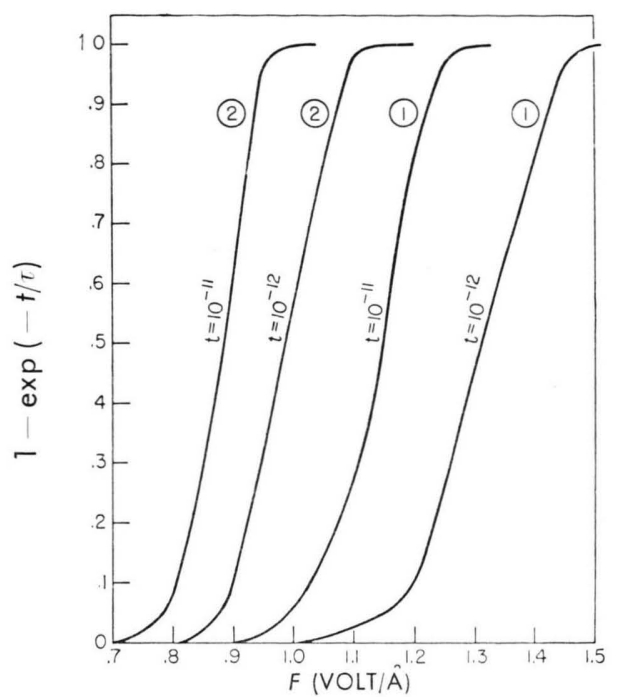

Abb. 5. $\left(1-e^{-t / \tau}\right)$ als Funktion der Feldstärke $F$ für zwei Werte von $t, t$ Verweilzeit der Atome im Feld. $\tau$ Halbwertszeit der Ionisierung bei Feldstärke $F$. Die mit (1) bezeichneten Kurven beziehen sich auf freie Atome, die mit (2) be-

zeichneten auf Atome nahe einer Wolframoberfläche.

Wir sind nun in der Lage, die Ionenprofile, die man bei Wasserstoffatomen erhalten sollte, als Funktion der Feldstärke zu berechnen. Abb. 5 zeigt
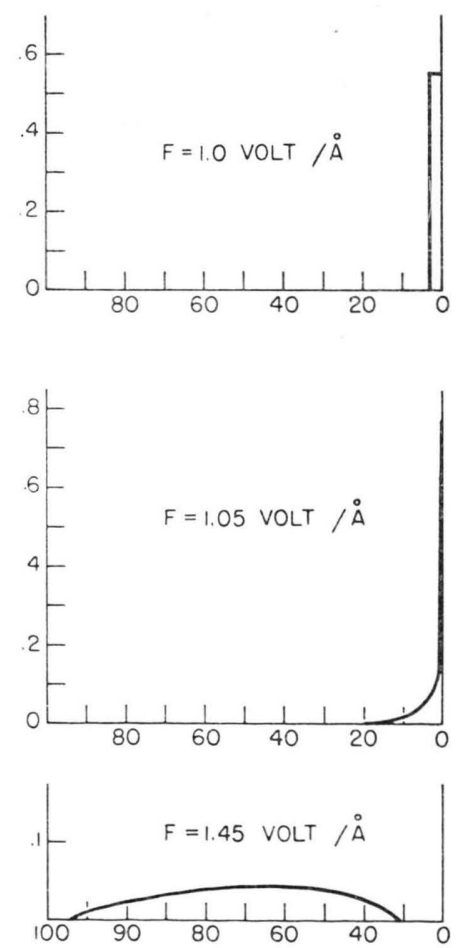

Abb. 6. Berechnete Profile als Funktion der Feldstärke für den theoretischen Fall des Wasserstoffatoms. $\left(1-e^{-t / \tau}\right)$ für zwei Werte von $t$. Es ist offensichtlich, daß Ionisation in der Nähe der Spitze bei niedrigeren Werten der Feldstärke vorkommt als im freien Raum. Man kann die theoretischen Profile aus Kurven wie denjenigen der Abb. 5 erhalten, indem man Gl. (6) anwendet. Solche Profile sind in Abb. 6 gezeichnet. Man sieht, daß sie den gemessenen (vgl. Abb. 4) sehr ähnlich sind. Die Verschiebung des Maximums und der vorzeitige Abfall bei sehr hohen Feldstärken ist darauf zurückzuführen, daß alle eintreffenden Teilchen schon vor Erreichen der Spitze ionisiert werden. Dieser Effekt wurde bei der Berechnung der Kurven von Abb. 6 berücksichtigt.

\section{Chemisorption}

Man findet manchmal, daß ein oder mehrere Massen-Profile in einem Spektrum keine Erweiterung mit ansteigendem Feld zeigen. Das weist auf einen festen Ionisationsort hin, der wahrscheinlich auf der Spitzenoberfläche selbst liegt. Abb. 7 zeigt die Hauptprofile im Feldionenspektrum des Methylalkohols. Man sieht, daß das Mutterion $\mathrm{CH}_{3} \mathrm{OH}^{+}$die typische Feldverbreitung zeigt, die von schwach oder gar nicht adsorbierten Teilchen zu erwarten ist, während das $\mathrm{CH}_{3} \mathrm{O}^{+}$-Ion diese nicht aufweist. Diese Tatsache deutet darauf hin, daß das letztere in der chemisorbierten Schicht entsteht und das Hauptprodukt der dissoziierenden Adsorption des Methanols auf einer

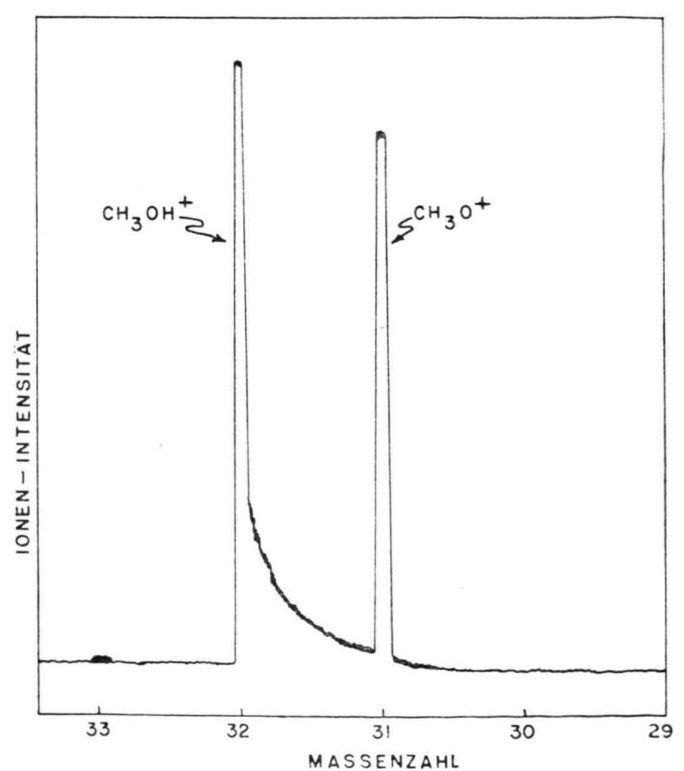

Abb. 7. Feldionenspektrum des Methanols bei mittlerer Feldstärke mit Verbreiterung des Mutterprofils. 
mit Sauerstoff bedeckten Wolframoberfläche ist. Die Müllerschen Versuche ${ }^{1}$ mit $\mathrm{Ba}$ usw. zeigen, daß solch ein Phänomen zumindest möglich ist. Diese Annahme wird von den folgenden Beobachtungen gestützt: (1) das Verhältnis von $\mathrm{CH}_{3} \mathrm{O}^{+}$zu $\mathrm{CH}_{3} \mathrm{OH}^{+}$ steigt mit fallendem Methanoldruck, was einer stärkeren Bindung des $\mathrm{CH}_{3} \mathrm{O}$ an der Oberfläche entspricht. (2) Es besteht ein kritisches Abreißfeld, das noch besprochen werden wird, wie Abb. 8 zeigt. Man sieht, daß das $\mathrm{CH}_{3} \mathrm{O}^{+}$-Ion erst bei höherer Feldstärke erscheint als das $\mathrm{CH}_{3} \mathrm{OH}^{+}$-Ion und schärfer einsetzt. Die große Ähnlichkeit zwischen der „appearance curve" für $\mathrm{CH}_{3} \mathrm{OH}^{+}$und den Kurven, die in Abb. 5 gezeichnet sind, ist offensichtlich.

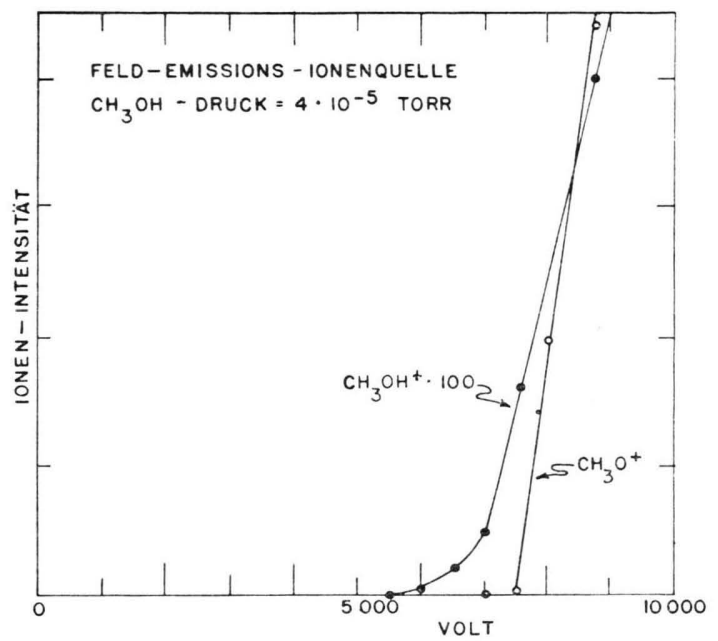

Abb. 8. Ionenströme bei Methanol als Funktion der Spannung.

\section{Impuls-Betrieb}

Die starke Adsorption von $\mathrm{CH}_{3}$ wird noch überzeugender vom folgenden Versuch gezeigt: Wenn bei der Chemisorption von Methanol ein Wasserstoffatom abgespalten wird, so daß die adsorbierte Einheit $\left(\mathrm{CH}_{3} \mathrm{O}\right)_{\mathrm{T}}$ ist, dann gilt

$$
\left(\mathrm{CH}_{3} \mathrm{O}\right)_{\mathrm{T}} \gg\left(\mathrm{CH}_{3} \mathrm{OH}\right)_{\mathrm{g}}, \Theta \gtrsim 0,1
$$

bei einem Druck von einigen $10^{-3}$ Torr. $\left(\mathrm{CH}_{3} \mathrm{OH}\right)_{\mathrm{g}}$ entspricht der Anzahl von Methanolmolekeln, die sich in dem Teil des Gasraums befinden, in dem das Feld wirksam ist. $\Theta$ bedeutet hier den Anteil der bedeckten Adsorptionsstellen.

Wenn nun das Feld für nur ganz kurze Zeiten (ungefähr $1 \mu \mathrm{sec}$ ) angeschaltet wird, so kann auch die Zahl der in dieser Zeit zur Spitze diffundierenden Molekeln ganz klein gemacht werden verglichen mit $\left(\mathrm{CH}_{3} \mathrm{O}\right)_{\mathrm{T}}$. Bei hoher Impulsfrequenz ist die Situation ähnlich wie bei konstantem Feld. Wird die Frequenz aber stark erniedrigt, so strebt das Verhältnis von $\mathrm{CH}_{3} \mathrm{O}^{+}$zu $\mathrm{CH}_{3} \mathrm{OH}^{+}$gegen unendlich. Abb. 9 zeigt, daß das auch der Fall ist. Es ist zu bemerken, daß die Impulsmethode nur dann anwendbar ist, wenn die Halbwertszeit der Ionisierung eines adsorbierten Teilchens ungefähr ebenso groß oder größer ist als die Zeit, in der ein Spannungspuls seinen Vollwert erreicht („rise time“), da sonst die Ionenenergie nicht der Beschleunigungsspannung $E_{1}$ entsprechen würde.

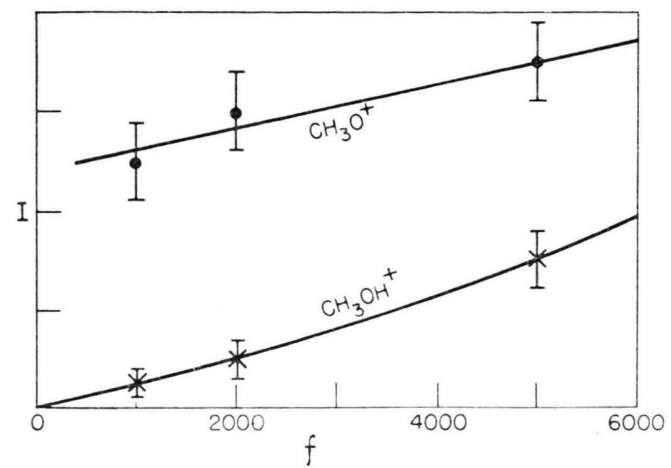

Abb. 9. $\mathrm{CH}_{3} \mathrm{O}$ - und $\mathrm{CH}_{3} \mathrm{OH}$-Ionenstrom als Funktion der Pulsfrequenz $f$ in $\sec ^{-1}$ für Pulse von $2 \mu$ sec Dauer.

\section{Mechanismus der Ionisation bei Chemisorption}

Es lohnt sich, einige Betrachtungen über den Mechanismus der Feldionisation adsorbierter Teilchen anzustellen. Nehmen zwei Elektronen an der Bindung teil, so spielt sich der Vorgang wahrscheinlich in zwei Schritten ab. Zuerst durchdringt eines der bindenden Elektronen die Potentialschwelle zwischen adsorbiertem Teilchen und Metall, so daß ein neutrales, aber polarisiertes Atom oder Molekül vor der Oberfläche zurückbleibt. Das letztere kann dann auf "gewöhnliche“ Weise vom Feld ionisiert werden. Die Entfernung von der Oberfläche, bei welcher der erste Schritt stattfinden muß, ist bei Adsorption nicht willkürlich gegeben oder aus Gl. (14) zu ermitteln, da die bindenden Elektronen im Durchschnitt auf den mittleren Bindungsabstand beschränkt, also ca. $1 \AA$ von der Oberfläche entfernt sind. Daher muß die Feldstärke mindestens genügend hoch sein, um diese Elektronen bis zum Fermi-Niveau des Metalls zu heben. Sie ist durch

$$
F_{\text {min }}=2 H / b
$$

gegeben. $b$ bedeutet hier den Bindungsabstand und $H$ die Bildungswärme für ein neutrales, aber polari- 
siertes Teilchen. Hat einmal das erste Elektron das Metall erreicht, so können zwei verschiedene Vorgänge stattfinden. Ist das Ionisierungspotential IP des neutralen Teilchens nicht viel größer als $H$, so wird das Atom oder Molekül durch Schwingungen auf eine solche Entfernung gehen, daß es nach Gl. (14) ionisiert wird. Ist das IP $\gg H$, so folgt dem ersten Schritt die Abdampfung eines neutralen Teilchens. Beide Möglichkeiten für den zweiten Schritt sind dadurch bedingt, daß die Kernschwingungsenergie des Gesamtteilchens die Dipol- und Polarisierungsenergie $E_{\mathrm{f}}$ überschreitet. Diese Tatsache ist wahrscheinlich für die relativ lange Lebensdauer von $\mathrm{CH}_{3} \mathrm{O}$ verantwortlich.

\section{Diskussion der Einzelresultate}

Die in Tab. 2 angegebenen Resultate werden jetzt ausführlicher besprochen.

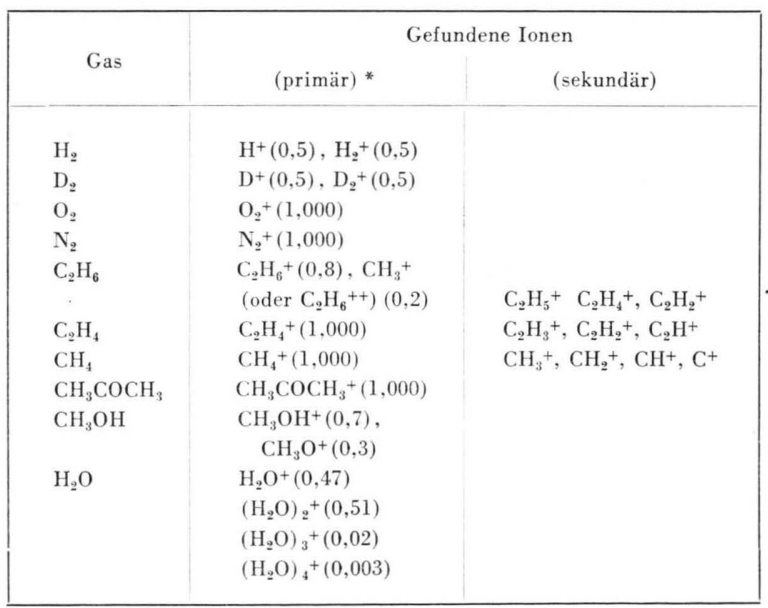

Tab. 2. Zusammenfassung der Ionenausbeute von einigen Gasen mit einer Wolframstütze bei Feldstärken von $1-2 \mathrm{~V} / \mathrm{A}$.

* Der Einfachheit halber sind isotope Ionen nicht angedeutet. Diese entsprechen den bekannten Häufigkeiten.

Wasserstoff. Abb. 10 zeigt Ionenströme von $\mathrm{H}^{+}$ und $\mathrm{H}_{2}{ }^{+}$als Funktion der Spannung. Man sieht, daß beide Ionenarten Feldverbreiterung zeigen, so daß Gasionisierung stattgefunden haben muß. Vorversuche mit pulsierendem Feld weisen darauf hin, daß $\mathrm{H}_{2}$ schwach, $\mathrm{H}$ gar nicht adsorbiert ist, so daß die $\mathrm{H}^{+}$. Ionen durch einen Sekundärprozeß entstehen müssen. Dieser besteht vielleicht aus dem Verlust eines Elektrons von $\mathrm{H}_{2}{ }^{+}$oder der Erzeugung von $\mathrm{H}_{2}{ }^{+}$in ab. stoßendem Zustand. Sind diese vorläufigen Resultate richtig, so hängen sie wahrscheinlich mit der Ver- unreinigung der Oberfläche zusammen. Es ist aber ferner zu bemerken, daß sich aus Gl. (15) und der Adsorptionswärme von Wasserstoff auf Wolfram ein minimales Abreißfeld von 3,4 Volt/ $\AA$ ergibt, bei welchem schon die meisten antreffenden $\mathrm{H}_{2}$-Molekeln vor der Spitze ionisiert werden.

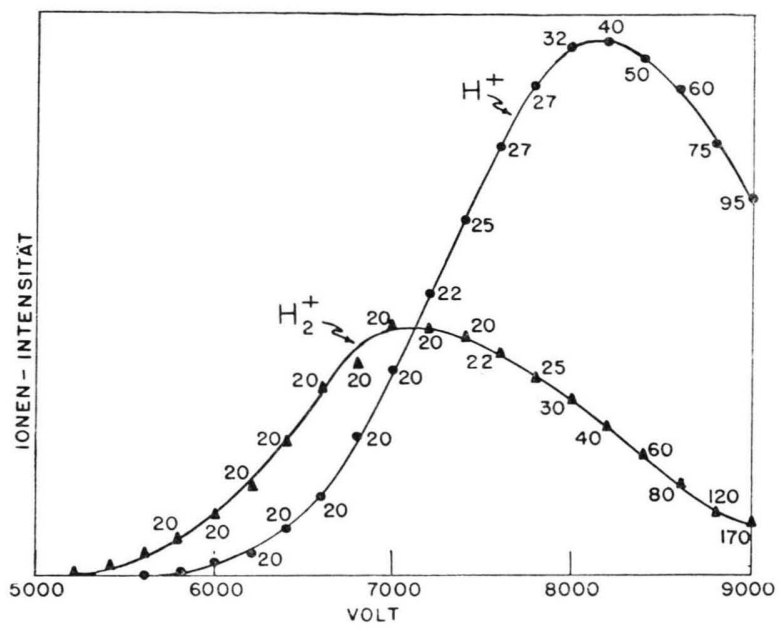

Abb. 10. Maximalwerte der Ionenströme bei Wasserstoff als Funktion der Spannung. Die Halbwerte der Verbreiterung sind durch die Zahlen neben den Punkten angegeben. Druck $3 \cdot 10^{-4}$ Torr.

Sauerstoff, Stickstoff. Bei Gleichfeld konnten nur $\mathrm{O}_{2}^{+}$und $\mathrm{N}_{2}^{+}$gefunden werden. Zumindest bei Sauerstoff ist das wahrscheinlich durch die sehr hohe Adsorptionswärme auf Wolfram zu erklären. Bei genügenden Feldstärken würden wahrscheinlich alle auftreffenden Sauerstoffmoleküle schon weit vor der Spitze ionisiert werden.

Kohlenwasserstoffe. Die bei Kohlenwasserstoffen gefundenen Spektren sind meist sehr einfach. Sekundärionen $(<0,001 \%)$ entsprechen dem Verlust eines oder mehrerer Wasserstoffatome. Die Beträge der meisten dieser Ionen steigen mit dem Quadrat des Druckes, und alle kommen scheinbar mit gebrochenen Massen vor, was bedeutet, daß sie durch $\mathrm{Zu}$ sammenstoß nach fast voller Beschleunigung entstanden sind. Dies überrascht nicht, da die mittlere freie Weglänge eines Ions viel größer ist als die Beschleunigungsdistanz. Die Wahrscheinlichkeit des Abreißens von einem oder mehreren Wasserstoffatomen ist nahezu von der Anzahl der abgerissenen Atome unabhängig, wenn die Ionenenergie 5000 bis $10000 \mathrm{eV}$ beträgt. Diese Erscheinung könnte durch Bremsung näher untersucht werden. Manchmal findet man Ionen, deren Intensität linear vom Druck 
abhängt, die aber doch gebrochene Massen zu haben scheinen. Es sind das metastabile Ionen, die noch vor der Beschleunigung durch Kernschwingung zerfallen.

Methanol. Die Hauptzüge dieses Spektrums sind schon erwähnt worden. Es ist nicht möglich, die Strukturformel des $\mathrm{CH}_{3} \mathrm{O}^{+}$-Ions anzugeben; doch sind Versuche mit $\mathrm{CD}_{3} \mathrm{OH}$ geplant, um diese zu ermitteln. Die Abwesenheit von $\mathrm{H}^{+}$-Ionen im Spektrum des Methanols hängt wahrscheinlich mit dem hohen IP des Wasserstoffs zusammen. Das Feld, bei dem $\mathrm{H}^{+}$und $\mathrm{H}_{2}^{+}$auftreten, ist beträchtlich gröBer als das für Methanol. Das Feld kann aber die Desorption von Wasserstoffatomen hervorrufen oder $\mathrm{H}_{2}$ kann desorbiert werden.

Wasser. Interessant ist hier der hohe Grad der Polymerisation. Da es kaum anzunehmen ist, daß Wasser beim Druck von $10^{-3}$ Torr vollkommen polymerisiert ist, muß die Erklärung in Gl. (3) gesucht werden. Die Energie $E_{\mathrm{f}}$ ist hier über $1 \mathrm{eV}$, und verursacht „künstliche“ physikalische Adsorption. Auf diese Weise entsteht eine mehrschichtige Bedekkung, die sehr flüssigkeitsähnlich ist und daher den hohen Polymerisationsgrad hervorruft. Bei niedriger Feldstärke zeigen die Massenprofile einige Struktur, was auf Energiedifferenzen hindeutet. Diese können durch Orientierungsunterschiede im Feld oder durch Ionisierung in verschiedenen Schichten hervorgerufen werden. Es ist zu hoffen, daß Versuche mit Edelgasen nahe dem wirklichen Kondensationspunkt dieses klarstellen werden.

\section{Mögliche Anwendungen}

Es gibt viele Anwendungsmöglichkeiten für die Verbindung von Feldionisation mit der Massenspektrometrie, von denen einige durch die vorliegenden Versuche angedeutet, viele aber noch nicht ausgenutzt worden sind.

(1) Untersuchung von bei der Chemisorption vorkommenden Fragmentierungen. Wie die Resultate mit Methanol zeigen, verspricht die Methode mit Hilfe von Energieauflösung und pulsierendem Feld Einsicht in manche bei heterogener Katalyse wichtige Vorgänge. Glücklicherweise sind gerade relativ schwache Adsorptionsvorgänge am zugänglichsten und auch in der Katalyse am wichtigsten. Vom Verhalten der Ionenintensitäten als Funktion der Fre- quenz bei Pulsbetrieb sollte man Haftkoeffizienten auf einzelnen Kristallflächen abschätzen können. Pulsbetrieb ermöglicht auch die Bestimmung von Adsorptionsisothermen auf einzelnen Kristallflächen ohne die störenden Wirkungen des Gleichfeldes. Auch muß die Spitze nicht aus Wolfram sein.

(2) Analyse von Gasmischungen: Aus Tab. 2 ergibt sich der durchweg einfache Charakter der Spektren. Das kommt daher, daß der Tunneleffekt fast keine Kernschwingungen hervorruft, weshalb Zerfall sehr selten ist. Abb. 11 zeigt das Feldionenspektrum des Acetons. Dieses sollte mit dem Elektronenstoßspektrum verglichen werden, das 19 Maxima ungefähr gleicher Größe aufweist, um die Möglichkeiten der Methode auf diesem Gebiet richtig einzuschätzen. Da man sekundäre Ionen als solche erkennen kann, sollten Sekundärvorgänge bei der Analyse nicht stören.

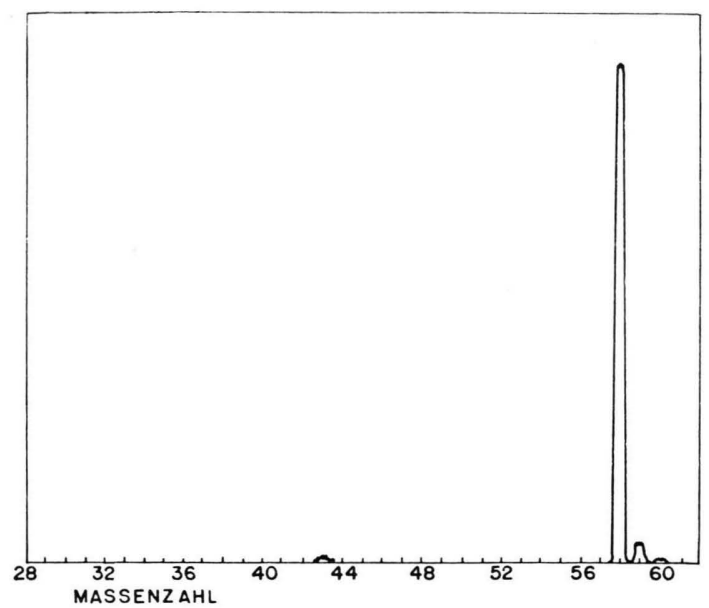

Abb. 11. Feldionenspektrum des Acetons, Druck $1 \cdot 10^{-4}$ Torr Hauptmaximum sowie isotope Maxima kommen nebst einem sehr schwachen $\mathrm{COCH}_{3}$-Maximum vor.

(3) Bestimmung kurzlebiger Zwischenprodukte und solcher von geringer Konzentration. Da störende Ionen aus der Muttersubstanz nicht oft vorkommen, kann man sehr geringe Mengen von Reaktionsprodukten nachweisen. Wie bei der gewöhnlichen Gasanalyse wäre da eine fokussierende Anordnung von Vorteil.

(4) Bestimmung der Halbwertszeit angeregter Teilchen. Da die Ionisierungswahrscheinlichkeiten vom IP abhängen, welches durch den spektroskopischen Zustand bedingt ist, sollte es möglich sein, Halbwertszeiten zu bestimmen, indem man Lichtstrahlen in verschiedenen Entfernungen von der 
Spitze einstellt und die Feldstärke so reguliert, daß nur angeregte Teilchen ionisiert werden.

(5) Analyse fester Adsorptionsschichten. Müllers Untersuchungen ${ }^{1}$ der Felddesorption aufgedampfter Metallschichten zeigen, daß die Methode zur chemischen Untersuchung fester Schichten verwendet werden kann.

(6) Erzeugung und Untersuchung negativer Ionen. Es wurde gefunden ${ }^{10}$, daß Zink-Phthalocyanin-Moleküle bei mittleren Feldstärken von negativ geladenen Spitzen abgerissen werden konnten, wenn der Bedeckungsgrad hoch war. Dieser Befund weist darauf hin, daß negative Ionen vom Felde erzeugt und mit einem Pulsfeld untersucht werden können.

Zum Abschluß wollen wir der $\mathrm{Nat}$ i o n a $\mathrm{l} \mathrm{S}$ cience F o und a tion, Washington, D. C., USA., und dem $\mathrm{Pe}$ t roleum Research Found der American Chemical Society für finanzielle Unterstützung unseren Dank aussprechen. Herr Dr. Peter $\mathrm{H}$ a a s en hat freundlicherweise unser Deutsch verbessert. Ihm möchten wir auch herzlich danken.

10 R. G o m e r, nicht veröffentlicht.

\title{
Der Astigmatismus des Toroidkondensators
}

\author{
Von H. Ewald und H. Liebl
}

\begin{abstract}
Aus dem Physikalischen Institut der Technischen Hochschule München
\end{abstract}
(Z. Naturforschg. 10 a, 872-876 [1955]; eingegangen am 5. Oktober 1955)

Herrn Professor Dr. J. Mat t a u c h zum 60. Geburtstag gewidmet

\begin{abstract}
Auf der Grundlage der ionenoptischen Berechnungen von Herzog, Marschall, Hachenberg und Svartholm werden die Abbildungseigenschaften eines Toroid-Sektorkondensators angegeben. Die experimentelle Erprobung eines solchen Kondensators ergab hinlängliche Übereinstimmung mit der Rechnung.
\end{abstract}

$\mathrm{D}$ er in der Massenspektrographie viel verwendete Zylinderkondensator ist ebenso wie der Kugelkondensator ein Spezialfall des allgemeineren Toroidkondensators. Die Sektorfeld-Elektroden eines Toroidkondensators (Abb. 1) haben Rotationsachse

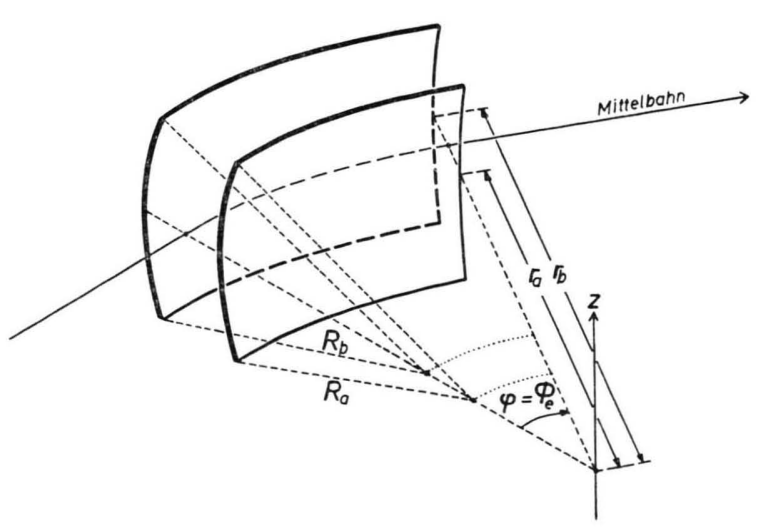

Abb. 1. Toroid-Kondensator.

(z-Achse eines $r, \varphi, z$-Zylinderkoordinaten-Systems) und Symmetrie-Ebene $(z=0)$ gemeinsam. Ihre radialen und axialen Hauptkrümmungsradien in den Punkten ihrer Schnittkreise mit der Ebene $z=0$ sind $r_{\mathrm{i}}, r_{\mathrm{b}}$, bzw. $R_{\mathrm{a}}, R_{\mathrm{b}}$. Die Mittelpunkte dieser Krümmungen fallen mit dem Nullpunkt des KoordinatenSystems zusammen bzw. sie liegen in der Ebene $z=0$ auf den Kreisen $r=\left|r_{\mathrm{a}}-R_{\mathrm{a}}\right|$ und $r=\left|r_{\mathrm{b}}-R_{\mathrm{b}}\right|$. $r_{\mathrm{a}}$ und $r_{\mathrm{b}}$ sind immer positiv. $R_{\mathrm{a}}$ und $R_{\mathrm{b}}$ sollen positiv oder negativ genommen werden, je nachdem ob die Schnittkreisbogen der Toroidsektoren mit Meridianebenen $\varphi=$ const der $z$-Achse ihre konkave oder konvexe Seite zuwenden.

Den Kreisbogen $r=a_{\mathrm{e}}\left(r_{\mathrm{a}}<a_{\mathrm{e}}<r_{\mathrm{b}}\right)$, $z=0$ zwischen beiden Elektroden nebst seinen geradlinigen Verlängerungen außerhalb des Sektorfeldes bezeichnen wir als Mittelbahn. Bei praktischen Ausführungen wird die Mittelbahn meist in der Mitte zwischen den Elektroden $\left(a_{\mathrm{e}}=\left(r_{\mathrm{a}}+r_{\mathrm{b}}\right) / 2\right)$ und außerdem $r_{\mathrm{b}}-r_{\mathrm{a}} \ll a_{\mathrm{e}}$ sein. Das Kondensatorfeld sei an seinen Rändern ideal begrenzt, es falle dort von seinen vollen Werten unstetig auf Null ab. Von der Wirkung der Streufelder sei also abgesehen.

Her z o g ${ }^{1}$ baute einen stigmatisch abbildenden, doppelfokussierenden Massenspektrographen unter Verwendung eines auch axial fokussierenden Kugelkondensators $\left(r_{\mathrm{a}}=R_{\mathrm{a}}, r_{\mathrm{b}}=R_{\mathrm{b}}\right)$. Um die stigmatische

${ }^{1}$ R. Herzog, Z. Naturforschg. 8a, 191 [1953]. 